Truck-based mobile wireless sensor networks for the experimental observation of vehicle-bridge interaction

This article has been downloaded from IOPscience. Please scroll down to see the full text article.

2011 Smart Mater. Struct. 20065009

(http://iopscience.iop.org/0964-1726/20/6/065009)

View the table of contents for this issue, or go to the journal homepage for more

Download details:

IP Address: 141.211.173.82

The article was downloaded on 06/04/2012 at 16:27

Please note that terms and conditions apply. 


\title{
Truck-based mobile wireless sensor networks for the experimental observation of vehicle-bridge interaction
}

\author{
Junhee Kim ${ }^{1}$, Jerome P Lynch ${ }^{1,2}$, Jong-Jae Lee ${ }^{3}$ and \\ Chang-Geun Lee ${ }^{4}$ \\ ${ }^{1}$ Department of Civil and Environmental Engineering, University of Michigan, Ann Arbor, \\ MI 48109, USA \\ 2 Department of Electrical Engineering and Computer Science, University of Michigan, \\ Ann Arbor, MI 48109, USA \\ ${ }^{3}$ Department of Civil and Environmental Engineering, Sejong University, Seoul 143-747, \\ Korea \\ ${ }^{4}$ Expressway and Transportation Research Institute, Korea Expressway Corporation, \\ Gyeonggi-do 445-812, Korea \\ E-mail: jerlynch@umich.edu
}

Received 4 November 2010, in final form 13 March 2011

Published 23 May 2011

Online at stacks.iop.org/SMS/20/065009

\begin{abstract}
Heavy vehicles driving over a bridge create a complex dynamic phenomenon known as vehicle-bridge interaction. In recent years, interest in vehicle-bridge interaction has grown because a deeper understanding of the phenomena can lead to improvements in bridge design methods while enhancing the accuracy of structural health monitoring techniques. The mobility of wireless sensors can be leveraged to directly monitor the dynamic coupling between the moving vehicle and the bridge. In this study, a mobile wireless sensor network is proposed for installation on a heavy truck to capture the vertical acceleration, horizontal acceleration and gyroscopic pitching of the truck as it crosses a bridge. The vehicle-based wireless monitoring system is designed to interact with a static, permanent wireless monitoring system installed on the bridge. Specifically, the mobile wireless sensors time-synchronize with the bridge's wireless sensors before transferring the vehicle response data. Vertical acceleration and gyroscopic pitching measurements of the vehicle are combined with bridge accelerations to create a time-synchronized vehicle-bridge response dataset. In addition to observing the vehicle vibrations, Kalman filtering is adopted to accurately track the vehicle position using the measured horizontal acceleration of the vehicle and positioning information derived from piezoelectric strip sensors installed on the bridge deck as part of the bridge monitoring system. Using the Geumdang Bridge (Korea), extensive field testing of the proposed vehicle-bridge wireless monitoring system is conducted. Experimental results verify the reliability of the wireless system and the accuracy of the vehicle positioning algorithm.
\end{abstract}

(Some figures in this article are in colour only in the electronic version)

\section{Introduction}

Vehicle-bridge interaction refers to the dynamic coupling that occurs between a vehicle and a bridge when the vehicle crosses at high speeds. A vehicle can be viewed as a large rigid mass (i.e. vehicle body) that is supported by vertical springdamper connections at each of its wheels. When crossing a bridge, the vehicle vertically vibrates and pitches, leading to the introduction of dynamic loading on the bridge. In turn, the vibrations of the bridge influence the dynamics of the vehicle. A complete understanding of this complex coupling between the vehicle and bridge is critical for fully understanding the dynamic behavior of bridges under live load conditions. In the design of highway bridges, vehicle-bridge interaction is 
accommodated through the use of dynamic impact factors. Dynamic impact factors allow the bridge designer to account for the dynamic response of a bridge when using an equivalent static load design methodology [1]. The current set of dynamic impact factors were obtained from finite element method (FEM) simulation of bridge systems in which the dynamics of the bridge, the behavior of the vehicle and the bridge road roughness have all been explicitly considered [8]. However, the limitation of FEM modeling renders the dynamic impact factors potentially inaccurate for some bridge designs [12]. To more accurately determine suitable dynamic impact factors, direct experimental observation of vehiclebridge interaction is necessary. There are many additional benefits that can be derived from experimental observation of vehicle-bridge interaction. For example, recent increases in the weight of heavy trucks has led to an acceleration of bridge deterioration [6]. Experimental observation of vehiclebridge interaction could lead to more accurate determination of bridge load capacities. Furthermore, experimental data would improve our understanding of vehicle-induced structural degradation.

Over the past two decades, powerful new sensor technologies have emerged including micro-electromechanical systems (or MEMS) [19, 20], wireless sensor networks $[17,21,11,32]$, guided wave sensors [23, 7] and fiber optic sensors [27, 2], just to name a few. Structural health monitoring (SHM) of bridge structures has served as the primary motivator for many of these developments. However, most of the sensors developed are designed to monitor structural response but not the loading inducing those responses. This is unfortunate because system identification and damage detection using output-only datasets are challenging inverse problems to solve when high uncertainty surrounds the bridge loading. Hence, the accuracy of current damage detection methods would be improved if the structural demand (i.e. the vehicle loading) was precisely known. Historically, weigh-in-motion systems (WIMS) have been used to observe the weight of vehicles crossing a bridge at a specific position. While WIMS can provide datasets from which bridge loads and their temporal variations can be modeled, such systems are inadequate for direct observation of vehicle-bridge interaction due to the fact that they only measure vehicle loading at a static location on the bridge. Another issue associated with WIMS is that their measurements contain significant uncertainty due to the temperature and time variation of their voltage outputs [31]. Alternatively, researchers have explored the use of video sensing to observe the motion of vehicles as they cross a bridge. For example, a piezoelectric-based WIMS can be replaced with a high-speed camera that can capture vehicles entering and leaving the view of the camera. Captured video can then be used to accurately assess the speed of the vehicle, the number of vehicle axles and the general class of vehicle (e.g. car, sport utility vehicle, truck, etc) through image processing [3]. While Chen et al [3] position their camera to view the road at a localized position, Fraser et al [4] alternatively propose the use of video cameras positioned to capture a view of an entire bridge. Using feature extraction and pattern recognition, vehicle types and position trajectories can be derived for all of the vehicles on the bridge. While a knowledge of average vehicle weight and trajectory can be used to model vehiclebridge interaction in a simulation environment, the actual interaction is not directly sensed in these video-based sensing systems.

The bridge and vehicle must both be instrumented to capture the dynamic coupling that exists during vehiclebridge interaction. In particular, instrumentation installed in the vehicle can provide data corresponding to the vehicle's vibratory response to the bridge as well as its position on the bridge as a function of time. When combined with response data collected from the bridge, a rich dataset corresponding to the system input (i.e. vehicle) and output (i.e. bridge) is created. The field has explored the monitoring of vehicles as they interact with roads, rail tracks and bridges. For example, Mizuno et al [18] propose the installation of MEMS accelerometers, global positioning system (GPS) receivers and a wired data acquisition system within railcars to capture their dynamic behavior as they travel over rail tracks. While the prototype system can measure the dynamic response of the vehicle, the system does not directly record the dynamic response of the rail or that of railway bridges. Even though bridges and vehicles have been independently monitored, monitoring both the vehicle and bridge within a single monitoring system architecture has not yet been attempted. This is due largely to the challenges a moving vehicle poses to tethered monitoring system architectures. Specifically, the mobility of the vehicle rules out the possibility of connecting vehicle sensors to the bridge monitoring system via coaxial wiring. Rather, two independent data acquisition systems would be needed, with one dedicated to monitoring the vehicle and the other monitoring the bridge. After collection, the vehicle and bridge response datasets would need to be combined with accurate time synchronization. In contrast, this study explores the use of wireless telemetry to eliminate the wires that limit the unification of vehicle-based mobile sensors within a bridge monitoring system.

In this study, wireless sensors are utilized as a building block of a comprehensive monitoring system designed for monitoring vehicle-bridge interaction. The work builds on recent research that has established the accuracy, reliability and cost-effectiveness of wireless monitoring systems for bridges [16, 21, 9, 32]. A wireless sensor network is proposed for installation in a heavy vehicle (i.e. truck) to record the dynamic response of the vehicle as it crosses a bridge instrumented with a permanent wireless monitoring system. The sensing transducers installed in the vehicle include accelerometers to measure vertical and horizontal acceleration and a gyroscope to capture the vehicle pitching motion. The bridge is instrumented with accelerometers to measure the vertical vibration of the bridge. Piezoelectric tactile sensors are also installed on the bridge road surface to sense the vehicle position. As the instrumented vehicle approaches the bridge, the permanent wireless monitoring system on the bridge establishes communication with the vehicle's mobile monitoring system to synchronize time and to initiate data collection. After the truck crosses the bridge, the wireless sensors on the truck wirelessly transmit their 


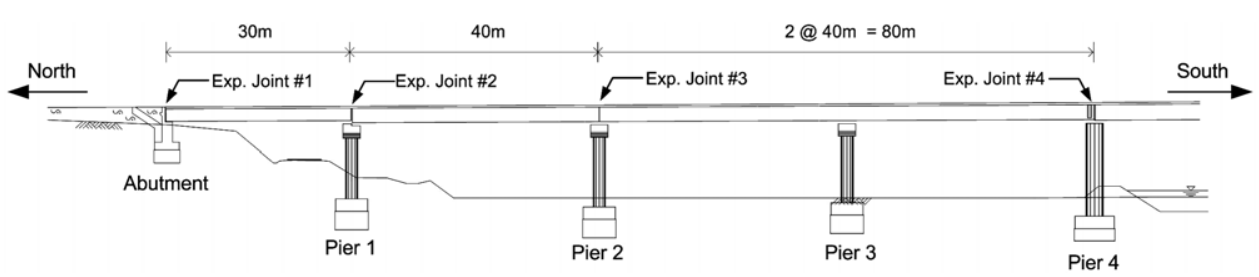

(a)

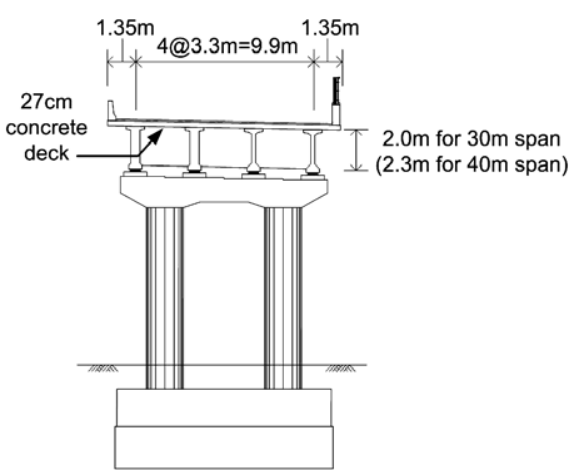

(b)

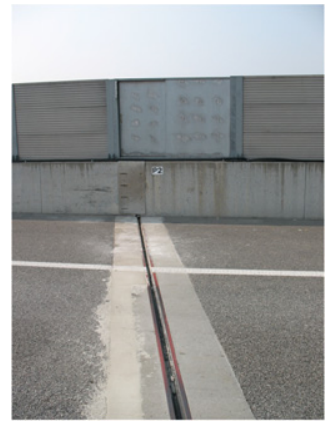

(c)

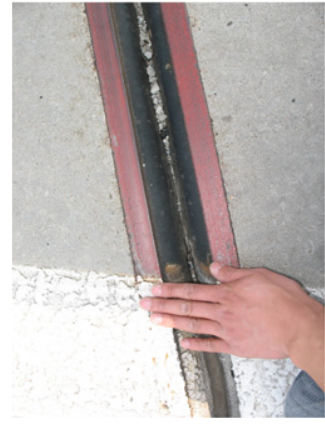

(d)

Figure 1. Geumdang Bridge (Icheon, Korea): (a) profile plan of the northernmost spans of the bridge; (b) cross-section profile; (c) expansion joint typical between adjacent spans and (d) close-up view of the third expansion joint.

synchronized response data to the bridge monitoring system where it is automatically combined with the bridge response data. The horizontal acceleration of the vehicle and the position information acquired from the piezoelectric tactile sensors are combined by the wireless monitoring system using Kalman filtering to estimate the vehicle trajectory. This paper begins with a description of the components of the proposed wireless monitoring system for experimentally observing vehicle-bridge interaction. Next, a theoretical description of the Kalman filter used to extract an accurate vehicle position trajectory is presented. Experimental validation of the proposed wireless monitoring system on the Geumdang Bridge (Icheon, Korea) is then presented. Finally, the paper concludes with a summary of the key project results and offers insight to the authors' future work aimed towards using the data collected by the wireless monitoring system for detailed analytical modeling of vehicle-bridge interaction.

\section{Overview of the wireless vehicle-bridge monitoring system}

\subsection{Geumdang Bridge}

In 2002, the Korea Expressway Corporation (KEX) completed construction of a redundant segment of the two-lane southbound Jungbu Inland Highway near Icheon, Korea [14]. The road segment was designed as an experimental test road with a dense array of sensors installed along its length (e.g. more than 1800 sensors along the $7.7 \mathrm{~km}$ road) to monitor the performance of the road pavement systems that are exposed to heavy truck loads. Along the length of the test road are three bridges: the Geumdang, Yeondae and Samseung bridges. Unlike the road, the three bridges are not monitored with a permanent set of sensors; rather, past work has monitored the bridges over short periods of time with wired and wireless monitoring systems [33]. This study selects the Geumdang Bridge (figure 1), a concrete bridge with a total length of $272 \mathrm{~m}$, to validate the performance of the proposed wireless monitoring system for vehicle-bridge interaction monitoring. Selection of the Geumdang Bridge is due to its length (i.e. the Yeondae and Samseung bridges are shorter) and for its straight planar alignment which makes tracking the moving vehicle easier.

The design of the Geumdang Bridge is very unique because it employs two different span types in its design. The northern half of the bridge spans $150 \mathrm{~m}$ and is constructed using four pre-cast concrete girders with a $27 \mathrm{~cm}$ thick concrete deck placed in composite action with the girders (figure 1(b)). The southern half of the bridge spans $122 \mathrm{~m}$ and is constructed as a continuous prestressed concrete box-girder. In 2006, the southern spans of the Geumdang Bridge were instrumented with an array of wireless sensors to measure the vertical acceleration of the bridge under traffic loads [16]. In this study, the northern span of the bridge will be instrumented with a wireless monitoring system to record the behavior of the bridge during loading by a test vehicle simultaneously monitored using wireless sensors. The northern portion of the bridge (figure 1(a)) is divided into three independent spans separated by expansion joints (figures 1(c) and (d)) that accommodate the thermal expansion of the bridge. The first span is $30 \mathrm{~m}$ long and is supported by the abutment structure and a pier. The second span is $40 \mathrm{~m}$ long and is supported by piers at its ends. The third span is $80 \mathrm{~m}$ long and is again supported by piers at its span ends; however, a redundant pier is placed midway along its length to offer additional support.

\subsection{Wireless sensors for long-range communication}

The building block of the proposed wireless vehicle-bridge monitoring system is the Narada wireless sensor node 


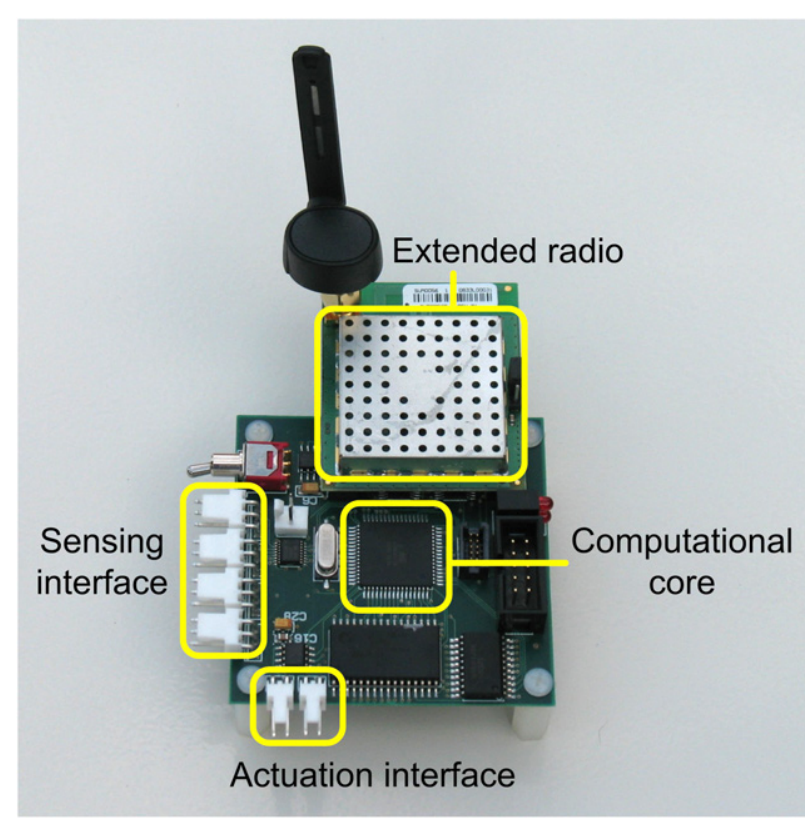

Figure 2. Narada wireless sensor node with key components highlighted.

(figure 2). Narada is a low-cost wireless sensor platform designed at the University of Michigan from commercial off-the-shelf embedded system components for use in civil infrastructure systems (e.g. bridges, buildings, dams, pipelines, etc) [28]. The data acquisition demands of the application have strongly shaped the design of Narada. For example, high-resolution digitization is needed to accurately record the low-level ambient vibrations common in civil engineering structures. Furthermore, the size of civil structures necessitates long-range communication between nodes (e.g. hundreds of meters). Finally, the overall cost of the wireless monitoring system must be low (e.g. less than $\$ 100$ per channel).

The Narada wireless sensor node is designed with a fourchannel, 16-bit analog-to-digital converter (ADC) to which a variety of sensors can be interfaced. The on-board ADC (Texas Instruments ADS8341) can sample sensor outputs spanning from 0 to $5 \mathrm{~V}$ on all four channels at rates as high as $100 \mathrm{kHz}$. The ADC is commanded by an 8-bit microcontroller (Atmel ATmega128) that operates at $8 \mathrm{MHz}$. The microcontroller contains flash memory $(128 \mathrm{kB})$ where embedded software is stored for automation of the wireless node operation (e.g. collect, process and communicate data). To accommodate the storage of sensor data on-board, an additional $128 \mathrm{kB}$ of static random access memory (SRAM) is integrated in the node design. For communication, the node includes the Texas Instruments CC2420, which is an IEEE802.15.4-compliant transceiver. The transceiver operates on the $2.4 \mathrm{GHz}$ wireless band which is readily accessible in most regions of the world. The data rate of the radio is $250 \mathrm{kbps}$ while its nominal range is $50-100 \mathrm{~m}$. The Narada node is designed with a power amplifier to boost the transceiver output power by $10 \mathrm{~dB}$, resulting in significant improvements in its communication range; for example, ranges in excess of $500 \mathrm{~m}$ have been reported [11]. The $500 \mathrm{~m}$ range is considered a conservative lower bound on the maximum communication range of the sensor, with higher ranges possible under favorable site conditions. The node is powered by a $6 \mathrm{~V}$ battery pack that is kept charged by solar panels. The total cost of the node is approximately $\$ 150$.

\subsection{Stationary wireless monitoring system on the bridge}

The $150 \mathrm{~m}$ northern span of the Geumdang Bridge is instrumented with a dense network of Narada wireless sensor nodes to which MEMS accelerometers and tactile sensors (i.e. piezoelectric strips) have been interfaced. As shown in figure 3, a total of 20 Narada wireless sensor nodes, each with an uniaxial MEMS accelerometer oriented in the vertical direction, is installed in the center lane of the bridge deck; the accelerometers are spaced equidistantly on each span. The Silicon Designs SD2012 accelerometer is selected for monitoring the vertical acceleration response of the bridge. While a large fraction of sensors for structural monitoring are single-ended, the SD2012 offers a low noise floor when utilized in differential output mode. The Narada ADC can accommodate differential sensor outputs by combining two of its available channels. In differential output mode, the sensitivity of the SD2012 accelerometer is $2 \mathrm{~V} \mathrm{~g}^{-1}$ while its noise floor is $13 \mu \mathrm{g} \mathrm{Hz}^{-1 / 2}$. The range of the accelerometer is $\pm 1 \mathrm{~g}$, which is ample for measuring bridge vibrations. Accelerometers are mounted on aluminum blocks that are bonded to the surface of the roadway by epoxy.

Tactile sensors are installed on the bridge deck to identify points in time when the truck drives over the sensors. The tactile sensors are designed from poly(vinylidene) fluoride, or PVDF, which is a piezoelectric polymeric material that exhibits a voltage change when dynamically strained. PVDF strips will capture in time when each axle of the truck drives over the strip. In this application, PVDF is selected because of its ductility and toughness which will give it great durability when being repeatedly driven over by heavy trucks. The tactile sensors are constructed from commercial PVDF sheets acquired from Measurement Specialties, Inc. The sheets are cut into $8 \mathrm{~mm}$ wide strips roughly $1.5 \mathrm{~m}$ long. Copper tape ( $5 \mathrm{~mm}$ wide) is

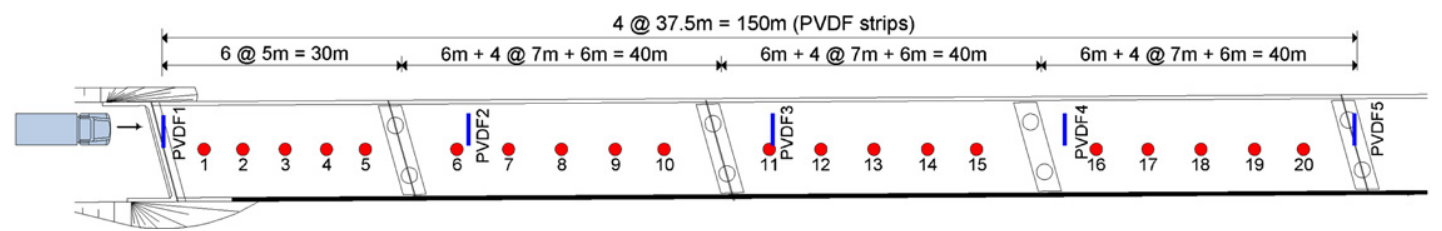

Figure 3. Stationary wireless monitoring system assembled from Narada wireless sensor nodes. Twenty (20) uniaxial accelerometers installed in the center of the bridge deck with five (5) PVDF tactile sensors installed at multiple locations along the bridge length. 


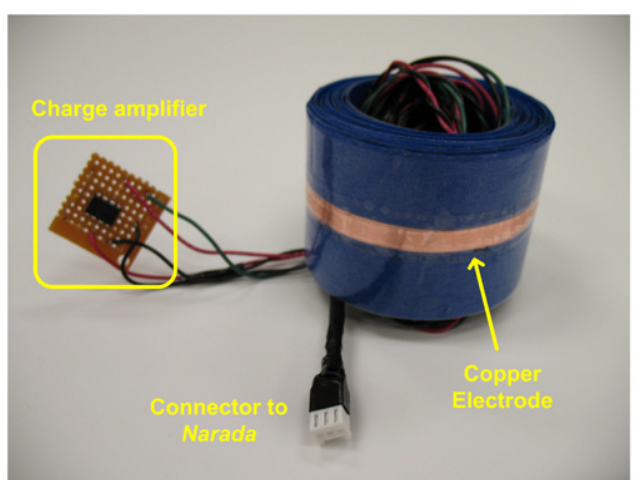

(a)

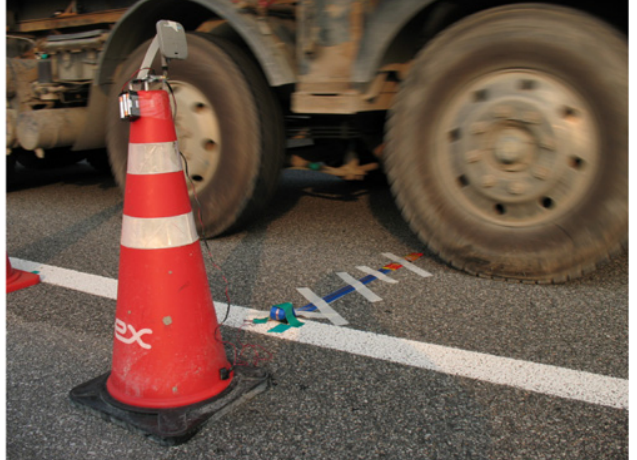

(b)

Figure 4. (a) PVDF tactile strip sensor rolled up prior to deployment on the bridge. Copper electrode and charge amplifier circuit clearly shown. (b) PVDF strip sensor installed on the bridge deck (at sensor location \#1) with a Narada wireless sensor shown on top of the orange traffic cone.

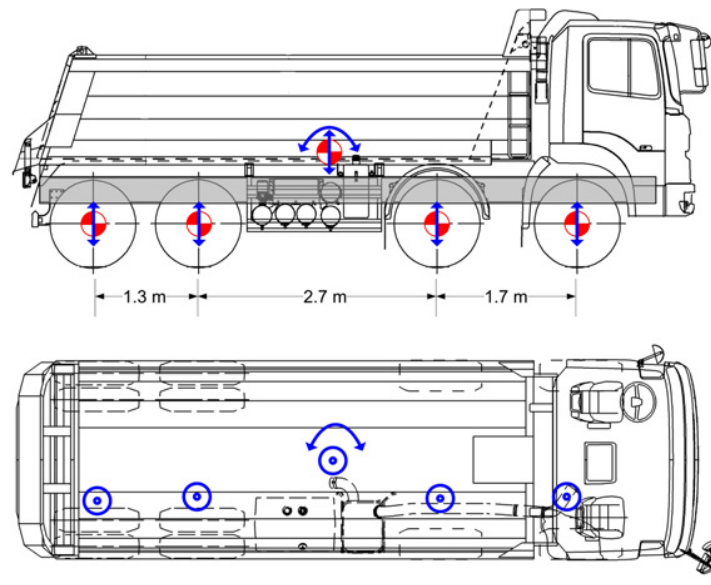

$\odot$ Vertical Accelerometer $\curvearrowright$ Pitching Angular Rate Gyro

(a)

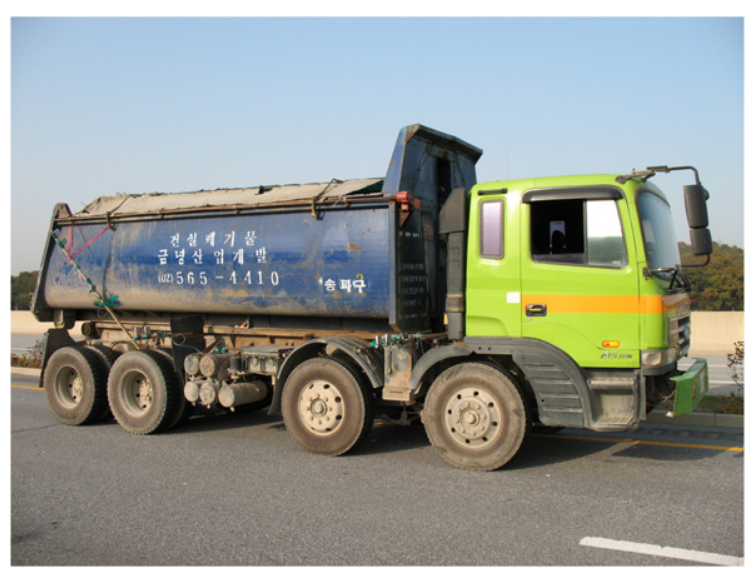

(b)

Figure 5. Experimental four-axle 20 ton truck with instrumentation. (a) Pitch-plane model of the truck with 6 DOFs identified for one sprung mass and four unsprung masses. (b) Picture of the truck on the Geumdang Bridge.

bonded to the top and bottom surfaces of the PVDF strip to serve as electrodes (figure 4(a)). A charge amplifier circuit is integrated with each PVDF strip to amplify its voltage before being interfaced to a Narada. In total, five (5) PVDF strips interfaced to Narada nodes are bonded to the surface of the road (figure 4(b)); the locations of the PVDF tactile sensors are presented in figure 3 .

\subsection{Mobile wireless sensor instrumentation on the truck}

To dynamically load the Geumdang Bridge, a four-axle truck is used (figure 5). Prior to the truck's arrival at the bridge, the weight of the truck is measured at a local weigh station. The total weight of the truck is 20.9 tons with the front, second, third and back axles each taking 4.3, 8.0, 4.6 and 4.0 tons, respectively. The truck dynamics can be modeled using a pitch-plane model with multiple rigid bodies connected by spring-damper linkages [5]. First, the truck body is comprised of a single sprung mass supported by the vehicle suspension system. Second, the wheels, axles, brakes and steering knuckle are modeled as concentrated masses centered at each axle. In total, the dynamics of the vehicle are modeled using five lumped masses and six degrees-of-freedom (DOF): vertical translation of the truck body, rotation of the truck body and vertical translation of each axle. Finally, the rigid body motion of the vehicle is modeled by its horizontal translation.

To monitor the dynamics of the truck body (i.e. the sprung mass of the pitch-plane model), sensors are concentrated in the truck body's center of gravity to monitor vertical acceleration, horizontal acceleration and gyroscopic motion associated with truck pitching (figure 6(a)). To monitor the vertical acceleration of the truck body, the Crossbow CXL02 capacitive accelerometer is selected. The CXL02 has a dynamic range of $\pm 2 \mathrm{~g}$, a sensitivity of $1 \mathrm{~V} \mathrm{~g}^{-1}$, and a noise floor of $0.15 \mathrm{mg}$. A second CXL02 is utilized to monitor the horizontal acceleration of the vehicle; this acceleration data will be used for truck positioning. A MEMS angular rate gyroscope (Analog Devices ADXRS624) is also installed at the center of gravity to capture the pitching motion of the truck. The ADXRS624 has a dynamic range of $\pm 50^{\circ} \mathrm{s}^{-1}$ 


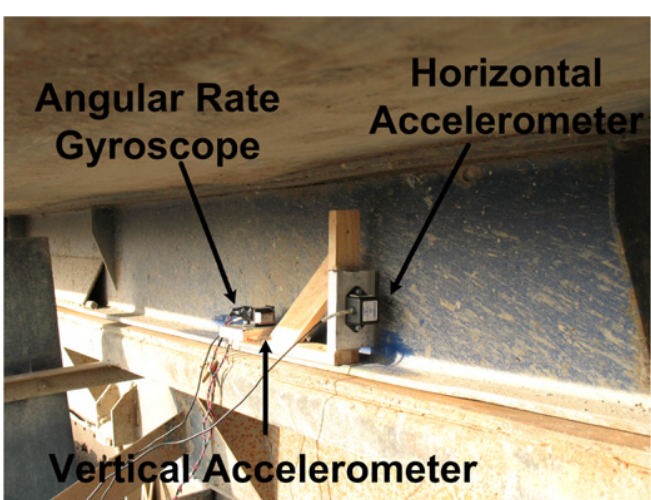

(a)

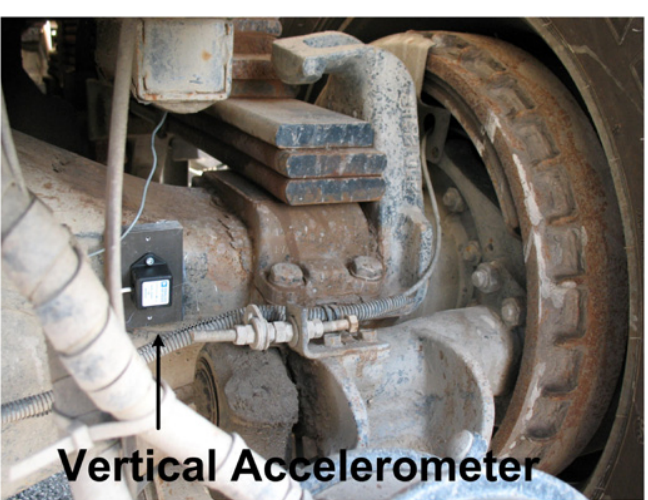

(b)

Figure 6. Installation of sensors on the experimental truck to monitor the 6 DOF associated with the truck pitch-plane model (figure 5(a)): (a) accelerometers and gyroscope installed at the truck body center of gravity and (b) accelerometer on the axle cover at the fourth (back) axle.

and a sensitivity of $25 \mathrm{mV} /{ }^{\circ} \mathrm{s}^{-1}$. Each axle of the truck is monitored using an accelerometer bonded to the axle oriented in the vertical direction. The Analog Devices ADXL105 accelerometer is selected for its high $\pm 10 \mathrm{~g}$ measurement range and low $2 \mathrm{mg}$ noise floor; the sensitivity of the ADXL105 is $250 \mathrm{mV} \mathrm{g}^{-1}$. The accelerometer is attached by epoxy to the underside of the leaf spring of each front axle. For the two back axles, the accelerometers are installed on the metallic cover of the axle (figure 6(b)). To record the sensor outputs, a Narada wireless sensor is interfaced with each sensing transducer installed on the truck. To enhance the performance of the wireless communications, the antennas of each wireless sensor are mounted to a $1.2 \mathrm{~m}$ wood post with the post installed in a near-vertical orientation $\left(70^{\circ}-75^{\circ}\right)$ on the passenger side of the truck.

\subsection{Operation of the wireless monitoring system during dynamic load testing}

The proposed wireless monitoring system for experimental observation of vehicle-bridge interaction is architecturally centralized with a central base station installed on the bridge. The base station consists of an IEEE802.15.4 receiver interfaced to a small single-board computer that coordinates the activity of the wireless monitoring system. The base station continuously transmits a beacon signal that can be received by a truck instrumented with Narada wireless sensors. Similarly, Narada sensors on the truck are designed to be in a receive-mode waiting to receive the beacon packet from the bridge base station. As the truck drives closer to the bridge (i.e. within $500 \mathrm{~m}$ of the bridge receiver), it will fall within communication range of the bridge monitoring system base station. Upon receipt of the beacon packet, the Narada wireless sensors on the truck acknowledge their existence by sending their identification numbers wirelessly back to the base station. As soon as the truck's wireless sensor nodes send their identification numbers, they enter a state ready to receive a second beacon packet from the bridge receiver to which their local clocks will be synchronized. Once the base station acknowledges that an instrumented truck is in the vicinity, it sends a command packet to the network of wireless sensor nodes on the bridge and truck commanding them to all synchronize their clocks to the arrival of the packet and to collect data for a set period of time at a specific sampling rate. This approach to time synchronization has been previously verified to be within $11 \mu \mathrm{s}$ [11], which is negligible when compared to the normal sample rates used in bridge monitoring (i.e. less than $1 \mathrm{kHz}$ ). After all of the sensors have collected their response data, they notify the base station that they are ready to transmit their response data back to the server. The server queries them one at a time for their data; the sensors on the truck are queried first because they may shortly be out of range. In this study, the base station commands the mobile and static wireless sensor nodes to collect $90 \mathrm{~s}$ of data at a sample rate of $100 \mathrm{~Hz}$. This time duration provides the monitoring system ample time to register the mobile wireless sensors, collect bridge and vehicle response data, and collect the data at the base station.

During experimental validation on the Geumdang Bridge, the KEX closes the test road to allow the four-axle truck unfettered access to the bridge. The base station and receiver (figure 7(a)) are installed on the shoulder of the southern end of the bridge. On site, the effective communication range of the base station is determined to be roughly $800 \mathrm{~m}$. While this is greater than past studies, the site conditions were favorable in this study for longer ranges. The 7 mobile Narada wireless sensors on the truck and the 25 static Narada wireless sensors on the bridge are set to communicate on the same wireless channel as the base station. Once the installation of the sensors on the truck and bridge is complete, the bridge is loaded by driving the truck across the bridge at three different velocities $\left(30,50\right.$ and $65 \mathrm{~km} \mathrm{~h}^{-1}$ ). The starting position of the truck is roughly $500 \mathrm{~m}$ up the road from the bridge. The truck is commanded by the researchers on site to accelerate until it achieves the desired test speed at which time the truck driver holds the velocity of the truck constant. Once the truck leaves the bridge, the truck driver brings the truck to a stop roughly $200 \mathrm{~m}$ past the southern bridge abutment. The truck is driven in the leftmost lane to ensure it drives over the PVDF tactile sensors installed on the road deck. 


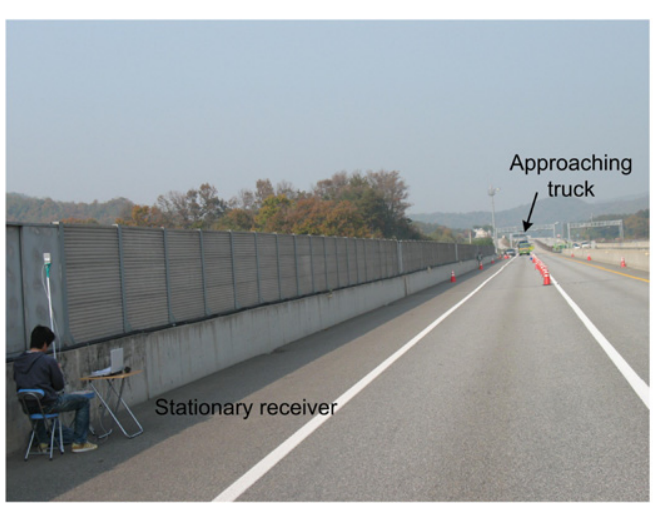

(a)

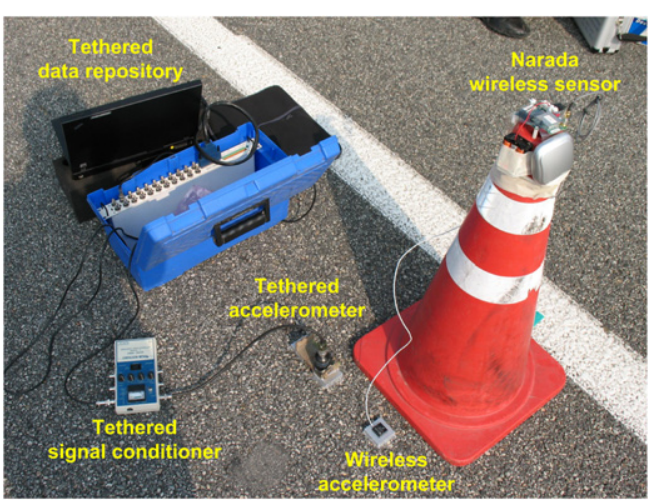

(b)

Figure 7. Dynamic load testing by wireless sensor networks. (a) Base station at the start of a test with the truck north of the bridge.

(b) Wireless accelerometer on the bridge deck side-by-side with a tethered piezoelectric accelerometer at sensor location \#8 (see figure 3 ).

\section{Theory of trajectory estimation}

\subsection{Review of trajectory estimation and integration of acceleration}

During the analysis of vehicle-bridge interaction analysis, accurate positioning of the vehicle provides the location of the dynamic load imposed on the bridge by the vehicle. Many trajectory estimation algorithms are available from the geodetic and navigation fields that integrate inertial measurements derived from accelerometers and gyroscopes to track bodies in space [13]. Closely related to inertial navigation is the work conducted in the civil engineering field in determining the displacement of structures based on their measured acceleration responses. For example, Lee et al [15] have proposed an acceleration-based low-pass filtering algorithm to derive the displacement of low-frequency-dominant structures using acceleration measurements. While the method works well for structural responses dominated by sinusoidal motion, it is not capable of reconstructing the pseudo-static response common during vehicle loading. In contrast, Smyth et al [25] propose a novel data fusion algorithm that combines acceleration and displacement measurements to overcome the challenge of low-frequency noise that is amplified when numerically integrating an acceleration signal.

Similar to Smyth et al [25], this study takes a data fusion approach to positioning an instrumented vehicle on a bridge by combining acceleration measurements of the vehicle with position information obtained from PVDF tactile sensors installed on the bridge deck. The proposed trajectory estimation algorithm explicitly considers measurement bias to account for sensor bias (e.g. non-zero offsets) and the tilting of the sensor during installation, among other biasing factors. Each sensor used has a unique bias that must be accounted for to achieve high precision inertial sensing [22, 34]. Kalman filtering and interval smoothing is then used to yield a highly accurate estimate of the vehicle location on the bridge.

\subsection{Tracking model formulation}

A mathematical model for vehicle tracking begins with consideration of a vehicle in which an accelerometer is installed at the center of gravity to measure horizontal acceleration. The one-dimensional trajectory of the horizontally moving vehicle is

$$
\begin{array}{cc}
\ddot{\bar{x}}(t)=\ddot{x}(t)-\delta \ddot{x}(t) \quad \text { with initial conditions } \bar{x}(0) \\
\text { and } \quad \dot{\bar{x}}(0)
\end{array}
$$

where $\ddot{\bar{x}}(t)$ is the true horizontal acceleration of the vehicle and $\ddot{x}(t)$ is the measured horizontal acceleration. Hence, $\delta \ddot{x}(t)$ corresponds to the acceleration measurement error. The measurement error, $\delta \ddot{x}(t)$, consists of a deterministic unknown bias, $b(t)$, and stochastic process noise, $w(t)$, as follows:

$$
\delta \ddot{x}(t)=b(t)+w(t) .
$$

By numerically integrating equation (1) in the discretetime domain, the true horizontal velocity, $\dot{\bar{x}}(t)$, and position, $\bar{x}(t)$, of the vehicle can be calculated:

$$
\begin{gathered}
\dot{\bar{x}}(k+1)=\dot{\bar{x}}(k)+(\ddot{x}(k)-\delta \ddot{x}(k)) \Delta t \\
\bar{x}(k+1)=\bar{x}(k)+\dot{\bar{x}}(k) \Delta t+0.5(\ddot{x}(k)-\delta \ddot{x}(k)) \Delta t^{2}
\end{gathered}
$$

where $\Delta t$ is the time step. The problem of estimating the position and velocity of the vehicle is confined to estimating the deterministic acceleration measurement error of $\delta \ddot{x}(t)$ (i.e. the bias, $b(k))$ and to treat the stochastic measurement error of $\delta \ddot{x}(t)$ as Gaussian noise, $w(k)$. A model-based data fusion approach that combines the horizontal acceleration of the vehicle with position, $x$, and velocity measurements, $\dot{x}$, obtained from PVDF tactile sensors is adopted. A state-space model that captures the evolution of the measurement error is proposed. Towards this end, a state vector, $\mathbf{z}$, is introduced as

$$
\mathbf{z}(t)=[\delta \dot{x}(t) \delta x(t) b(t)]^{\mathrm{T}}
$$

where $\delta \dot{x}$ and $\delta x$ are the velocity and position measurement errors, respectively. Based on equations (2) and (5), a continuous-time state-space equation can be written to model the measurement error in the system:

$$
\dot{\mathbf{z}}(t)=\left[\begin{array}{lll}
0 & 0 & 1 \\
1 & 0 & 0 \\
0 & 0 & 0
\end{array}\right] \mathbf{z}(t)+\left[\begin{array}{l}
1 \\
0 \\
0
\end{array}\right] w(t)=\mathbf{A z}(t)+\mathbf{w}(t) .
$$


Equation (6) is converted to a discrete-time state-space representation:

$$
\mathbf{z}(k+1)=\mathbf{A}_{\mathrm{d}} \mathbf{z}(k)+\mathbf{w}(k)
$$

where $\mathbf{A}_{\mathrm{d}}$ is the state transition matrix and determined as

$$
\mathbf{A}_{\mathrm{d}}=\mathrm{e}^{\mathrm{A} \Delta t}=\left[\begin{array}{ccc}
1 & 0 & \Delta t \\
\Delta t & 1 & 0.5 \Delta t^{2} \\
0 & 0 & 1
\end{array}\right]
$$

In order to consider the uncertainty of acceleration measurement, process noise, $\mathbf{w}(k)$, in equation (7) is considered as

$$
\mathbf{w}(k)=\left[\begin{array}{c}
N(0, Q(k)) \\
0 \\
0
\end{array}\right]
$$

When additional measurements are available from the PVDF tactile sensors (i.e. vehicle position and velocity), the measurement error in the vehicle position and velocity can be captured in the observation of the state:

$$
\mathbf{y}(k)=\left[\begin{array}{lll}
1 & 0 & 0 \\
0 & 1 & 0
\end{array}\right] \mathbf{z}(k)+\mathbf{v}(k)=\mathbf{C}_{\mathbf{d}} \mathbf{z}(k)+\mathbf{v}(k)
$$

where process noise, $\mathbf{v}(k)$, is also included in the observation model to capture the uncertainty in the measured vehicle position and velocity as measured by the PVDF tactile sensors. Here, $\mathbf{v}(k)$ is considered as

$$
\mathbf{v}(k)=\left[\begin{array}{c}
N\left(0, R_{1}(k)\right) \\
N\left(0, R_{2}(k)\right)
\end{array}\right] .
$$

\subsection{Data fusion by Kalman filtering}

Due to the assumption of stochastic Gaussian noise, Kalman filtering is ideally suited to extract an accurate estimate of the hidden states (i.e. velocity and position measurement errors and bias) of the system, $\mathbf{z}(k)$ [26]. The Kalman filter algorithm consists of two main stages: state/covariance prediction and correction.

3.3.1. Prediction stage. By equation (7), the a priori state, $\mathbf{z}(k+1 \mid k)$, and covariance, $\mathbf{P}(k+1 \mid k)$, are expressed, respectively, as

$$
\begin{gathered}
\mathbf{z}(k+1 \mid k)=\mathbf{A}_{\mathrm{d}} \mathbf{z}(k \mid k) \\
\mathbf{P}(k+1 \mid k)=\mathbf{A}_{\mathrm{d}} \mathbf{P}(k \mid k) \mathbf{A}_{\mathrm{d}}^{\mathrm{T}}+\mathbf{Q}
\end{gathered}
$$

where $\mathbf{Q}$ is a covariance matrix related to the uncertainty of the horizontal acceleration sensing in equation (9). Combining the numerically integrated vehicle velocity and location (i.e. equations (3) and (4)) and the estimated a priori states (equation (12)) of the measurement error, the velocity and location of the vehicle are calculated, respectively, as

$$
\begin{aligned}
& \dot{\hat{x}}(k+1)=\dot{\bar{x}}(k+1)-\delta \dot{x}(k+1 \mid k) \\
& \hat{x}(k+1)=\bar{x}(k+1)-\delta x(k+1 \mid k)
\end{aligned}
$$

3.3.2. Correction stage. Assume at the time step $k+1$, the second axle of the vehicle hits a PVDF tactile sensor. Based on the location of the PVDF sensor, the location of the vehicle, $x(k+1)$, is determined. Similarly, the velocity of the vehicle, $\dot{x}(k+1)$, is determined by the timing between the axle crossings as measured by the PVDF sensor. Then, the measurement errors are calculated and treated as the observed states:

$$
\left[\begin{array}{l}
\delta \dot{x}(k+1) \\
\delta x(k+1)
\end{array}\right]=\left[\begin{array}{l}
\dot{\bar{x}}(k+1)-\dot{x}(k+1) \\
\bar{x}(k+1)-x(k+1)
\end{array}\right]=\mathbf{y}(k+1) .
$$

The a posteriori state and covariance matrices can be calculated, respectively, as

$$
\begin{aligned}
& \mathbf{z}(k+1 \mid k+1)=\mathbf{z}(k+1 \mid k)+\mathbf{K}(k+1)(\mathbf{y}(k+1) \\
& \left.\quad-\mathbf{C}_{\mathrm{d}} \mathbf{z}(k+1 \mid k)\right) \\
& \mathbf{P}(k+1 \mid k+1)=\left(\mathbf{I}-\mathbf{K}(k+1) \mathbf{C}_{\mathrm{d}}\right) \mathbf{P}(k+1 \mid k)
\end{aligned}
$$

where the updated Kalman gain matrix, $\mathbf{K}(k+1)$ :

$$
\mathbf{K}(k+1)=\mathbf{P}(k+1 \mid k) \mathbf{C}_{\mathrm{d}}^{\mathrm{T}}\left(\mathbf{C}_{\mathrm{d}} \mathbf{P}(k+1 \mid k) \mathbf{C}_{\mathrm{d}}^{\mathrm{T}}+\mathbf{R}\right)^{-1} .
$$

In equation (19), $\mathbf{R}$ is a diagonal covariance matrix related to the uncertainty of the PVDF sensing in equation (11). Using the estimated a posteriori states of the measurement errors, the velocity and location of the vehicle are calculated, respectively, as

$$
\begin{aligned}
& \dot{\hat{x}}(k+1)=\dot{\bar{x}}(k+1)-\delta \dot{x}(k+1 \mid k+1) \\
& \hat{x}(k+1)=\bar{x}(k+1)-\delta x(k+1 \mid k+1) .
\end{aligned}
$$

\subsection{Fixed-interval smoothing}

To enhance the accuracy of the unique Kalman filter formulated, fixed-interval smoothing is adopted. Since the precise timing of when the vehicle enters and exits the bridge is known based on PVDF tactile sensors installed at the bridge end-points, fixed-interval smoothing [24] over the time period when the vehicle is on the bridge is prudent. The aforementioned Kalman filter is implemented off-line forwards in time (from the time when the vehicle enters the bridge) and backwards in time (from the time when the vehicle exits the bridge), resulting in two estimates for the system state: $\hat{\mathbf{z}}_{\mathrm{f}}(k)$ and $\hat{\mathbf{z}}_{\mathrm{b}}(k)$, respectively. The smoothing process derives a smoothed state, $\hat{\mathbf{z}}_{\mathrm{s}}(k)$, as

$$
\hat{\mathbf{z}}_{\mathrm{s}}(k)=\mathbf{K}_{\mathrm{f}}(k) \hat{\mathbf{z}}_{\mathrm{f}}(k)+\mathbf{K}_{\mathrm{b}}(k) \hat{\mathbf{z}}_{\mathrm{b}}(k)
$$

where $\mathbf{K}_{\mathrm{f}}(k)$ and $\mathbf{K}_{\mathrm{b}}(k)$ are coefficient matrices that weigh the relative contribution of the forward and backward state estimates. To guarantee unbiased state estimates, it requires that the sum of the coefficient matrices yield the identity matrix (i.e. $\mathbf{K}_{\mathrm{f}}(k)+\mathbf{K}_{\mathrm{b}}(k)=\mathbf{I}$ ). As a result, equation (22) can be further simplified as

$$
\hat{\mathbf{z}}_{\mathrm{s}}(k)=\mathbf{K}_{\mathrm{f}}(k) \hat{\mathbf{z}}_{\mathrm{f}}(k)+\left(\mathbf{I}-\mathbf{K}_{\mathrm{f}}(k)\right) \hat{\mathbf{z}}_{\mathrm{b}}(k) .
$$

The covariance matrix of the smoothed state, $\mathbf{P}_{\mathrm{s}}(k)$, can be calculated as

$$
\begin{aligned}
& \mathbf{P}_{\mathrm{s}}(k)=\operatorname{cov}\left(\hat{\mathbf{z}}_{\mathrm{s}}(k) \hat{\mathbf{z}}_{\mathrm{s}}^{\mathrm{T}}(k)\right)=\mathbf{K}_{\mathrm{f}}(k) \mathbf{P}_{\mathrm{f}}(k) \mathbf{K}_{\mathrm{f}}^{\mathrm{T}}(k) \\
& +\left(\mathbf{I}-\mathbf{K}_{\mathrm{f}}(k)\right) \mathbf{P}_{\mathrm{b}}(k)\left(\mathbf{I}-\mathbf{K}_{\mathrm{f}}(k)\right)^{\mathrm{T}}
\end{aligned}
$$



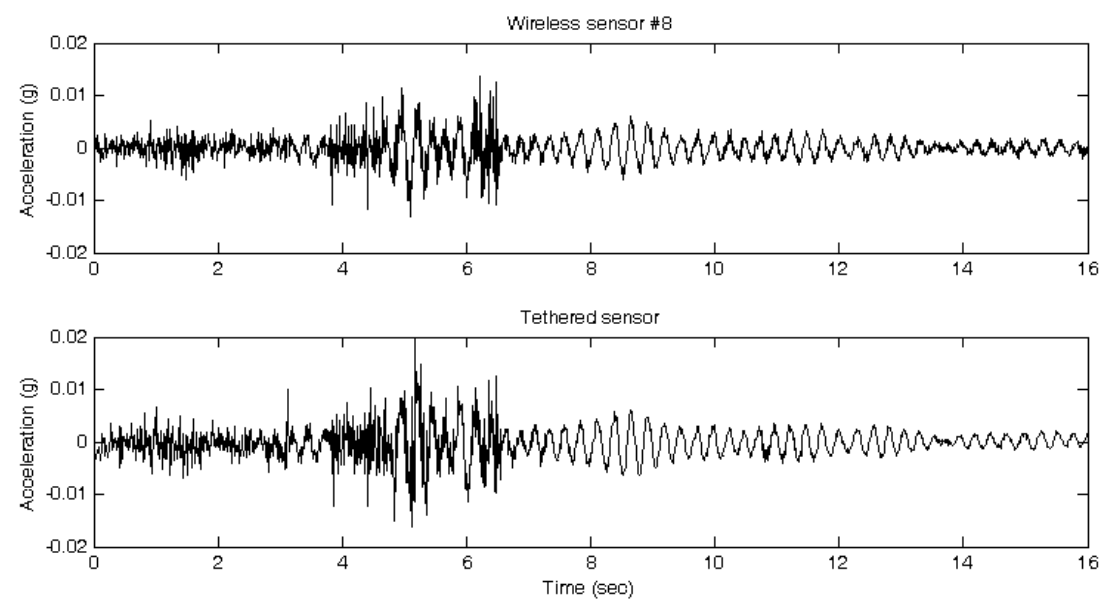

Figure 8. Comparison of the bridge vertical acceleration at sensor location \#8 (figure 3) as measured by the Narada wireless (top) and tethered accelerometers (bottom) when loaded by a 20.9 ton truck driving at $65 \mathrm{~km} \mathrm{~h}^{-1}$.

where $\mathbf{P}_{\mathrm{f}}(k)$ and $\mathbf{P}_{\mathrm{b}}(k)$ are the covariance matrices of the forward and backward Kalman filtering processes. Minimizing $\mathbf{P}_{\mathrm{s}}(k)$ with respect to $\mathbf{K}_{\mathrm{f}}(k)$ in equation (24) leads to a condition for an optimal forward coefficient matrix, $\mathbf{K}_{\mathrm{f}}(k)$ :

$$
\mathbf{K}_{\mathrm{f}}(k)=\mathbf{P}_{\mathrm{b}}(k)\left(\mathbf{P}_{\mathrm{f}}(k)+\mathbf{P}_{\mathrm{b}}(k)\right)^{-1} .
$$

Finally, by substituting the optimal forward coefficient matrix into equations (23) and (24), the smoothed state and its covariance matrix can be determined, respectively, as

$$
\begin{gathered}
\hat{\mathbf{z}}_{\mathrm{s}}(k)=\mathbf{P}_{\mathrm{b}}(k)\left(\mathbf{P}_{\mathrm{f}}(k)+\mathbf{P}_{\mathrm{b}}(k)\right)^{-1} \hat{\mathbf{z}}_{\mathrm{f}}(k) \\
+\mathbf{P}_{\mathrm{f}}(k)\left(\mathbf{P}_{\mathrm{f}}(k)+\mathbf{P}_{\mathrm{b}}(k)\right)^{-1} \hat{\mathbf{z}}_{\mathrm{b}}(k) \\
\mathbf{P}_{\mathrm{s}}(k)=\left(\mathbf{P}_{\mathrm{f}}^{-1}(k)+\mathbf{P}_{\mathrm{b}}^{-1}(k)\right)^{-1} .
\end{gathered}
$$

\section{Experimental validation}

\subsection{Accuracy of the wireless monitoring system}

The Narada wireless sensor has been extensively deployed on a large number of structures including bridges [11], wind turbines [29] and naval ships [30]. Similar to these past studies, the measurement accuracy of the Narada wireless sensors installed on the Geumdang Bridge are verified using a traditional tethered data acquisition system. A National Instruments 16-bit data acquisition system (Model 60362E) is used with a PCB Piezotronics 393B12 integrated circuit piezoelectric (ICP) accelerometer interfaced. The accelerometer measurement range is $\pm 0.5 \mathrm{~g}$ and its noise floor is $1.3 \mu \mathrm{g}(\mathrm{Hz})^{-1 / 2}$. The accelerometer is well suited for bridge monitoring because of its high sensitivity $\left(10 \mathrm{~V} \mathrm{~g}^{-1}\right)$. To provide a constant excitation to the ICP accelerometer and to amplify the accelerometer output by a factor of 10 , the PCB Piezotronics 480B21 signal conditioner is adopted. The tethered 393B12 ICP accelerometer is installed adjacent to the wireless accelerometer at sensor location \#8 (as denoted in figure 3). A picture of the side-by-side accelerometer installation is depicted in figure 7(b). Figure 8 presents the measured vertical acceleration of the bridge at sensor \#8 as measured by the Narada wireless and tethered systems.
The response measured corresponds to the test vehicle (i.e. the 20.9 ton truck) driving over the bridge at $65 \mathrm{~km} \mathrm{~h}^{-1}$. In general, both time-history responses are in excellent agreement. As expected, the Narada wireless sensor has a slightly elevated level of noise in the measured acceleration data due to the lower sensitivity and higher noise floor of the SD2012 accelerometer as compared to the PCB 393B12 accelerometer.

\subsection{Vehicle trajectory estimation}

The test vehicle is parked $600 \mathrm{~m}$ north of the Geumdang Bridge at the start of testing. After the wireless monitoring system initiates data collection using the mobile wireless sensors on the truck and the static wireless sensors on the bridge, the truck is commanded to accelerate to achieve a desired speed (e.g. $30 \mathrm{~km} \mathrm{~h}^{-1}$ ) before entering the bridge. After crossing the bridge, the truck decelerates until it comes to a stop approximately $200 \mathrm{~m}$ past the south end of the bridge. At the end of the test, the wireless monitoring system collects the horizontal acceleration of the vehicle as shown in figure 9(a). Based on the measured horizontal acceleration time history, the acceleration of the truck is zero at the start due to it being at rest. The truck accelerates with positive horizontal acceleration at $2.1 \mathrm{~s}$ until it achieves $30 \mathrm{~km} \mathrm{~h}^{-1}$ before entering the bridge at 26.9 s. During the time the truck is on the bridge (26.9$44.9 \mathrm{~s}$ ), the truck has a constant velocity and hence experiences zero horizontal acceleration. However, after exiting the bridge at $44.9 \mathrm{~s}$, the truck rapidly decelerates until it comes to a full stop at approximately $55 \mathrm{~s}$. In addition, the five PVDF tactile sensors on the bridge capture the time at which each axle of the truck rides over the sensor. Figure 9(b) superimposes the PVDF output voltage at all five PVDF tactile sensor locations. Each of the four truck axles are evident (e.g. four major voltage spikes) in the response measured at each PVDF tactile sensor. To determine the truck velocity, the spacing between the truck axles and the time of their crossing each PVDF tactile sensor is used.

The measured horizontal acceleration of the truck and the truck position and velocity (as measured at each of the 

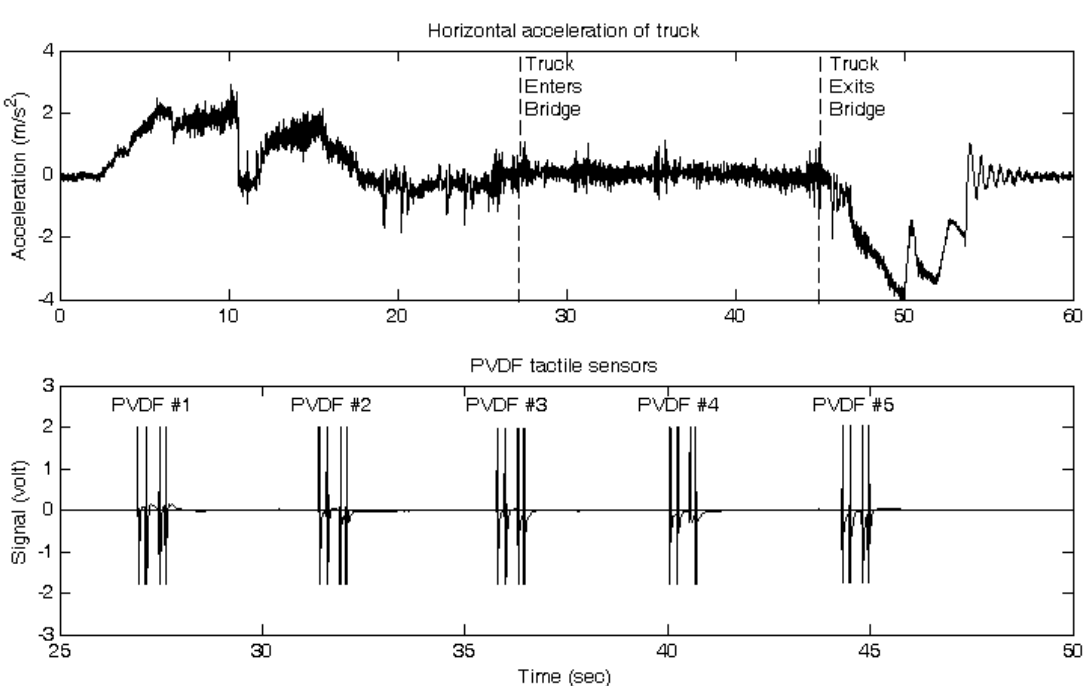

Figure 9. Trajectory sensing of the four-axle truck crossing the Geumdang Bridge at $30 \mathrm{~km} \mathrm{~h}^{-1}$. Horizontal acceleration time-history of the truck (top) and its position information measured by the five PVDF tactile sensors (bottom) with all four truck axles evident from the PVDF tactile sensor response.

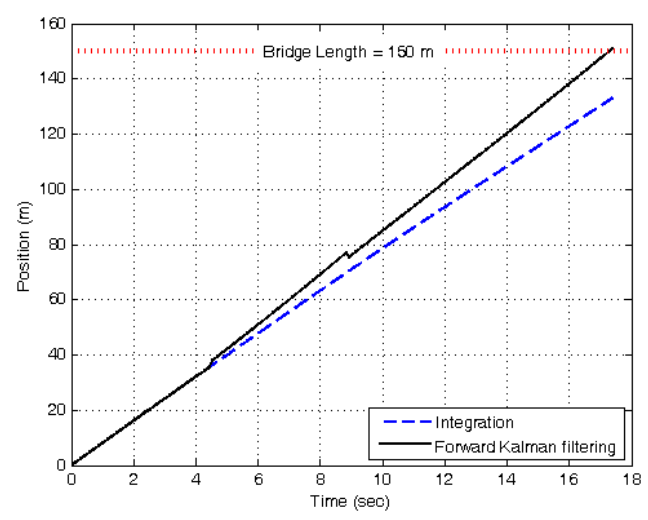

(a)
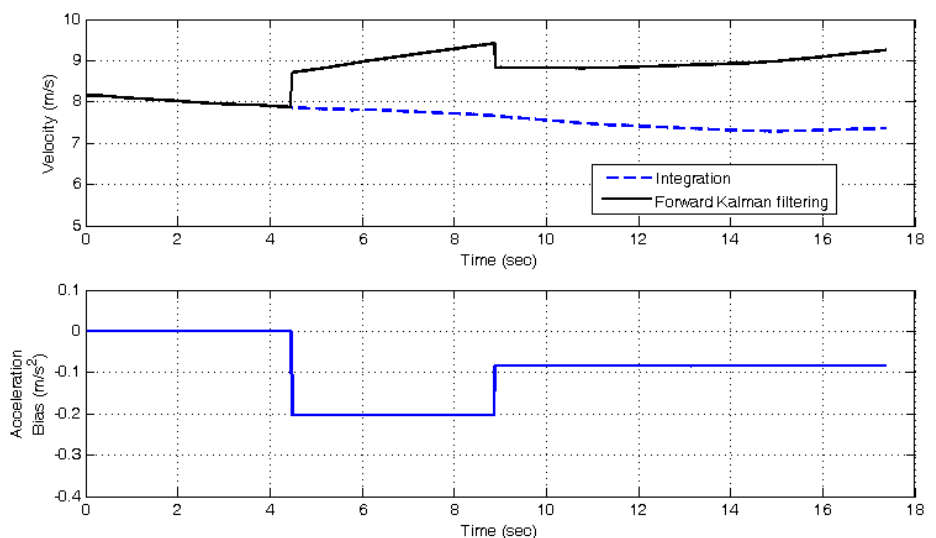

(b)

Figure 10. Results from the forward Kalman filter: (a) estimated truck trajectory along the $150 \mathrm{~m}$ bridge and (b) estimated velocity (top) and estimated accelerometer bias (bottom).

five PVDF tactile sensors) serve as inputs to the trajectory estimation algorithm previously derived (section 3). Based on the time the truck enters and exits the bridge (26.9 s and $44.9 \mathrm{~s}$, respectively), the Kalman filter is applied to the data in both a forward and backward manner. The output of the forward and backward Kalman filters are presented in figures 10 and 11, respectively. In each figure, the estimated truck position, velocity and acceleration bias are plotted over the $18 \mathrm{~s}$ the truck is on the bridge. In each figure, $0 \mathrm{~s}$ denotes the time when the first truck axle enters the bridge while $18 \mathrm{~s}$ denotes when the last axle exits the bridge. For both the forward and backward Kalman filters, the position of the truck at the end of the analysis is $150 \mathrm{~m}$ away from the start, which corresponds to the length of the bridge $(150 \mathrm{~m})$. In comparison, the position based on pure forward integration of the horizontal acceleration results in a final position of $132.9 \mathrm{~m}$ at the end of the test; this inaccurate position estimate corresponds to an error of $11.4 \%$. Similarly, backward Kalman filtering places the truck at $-1.3 \mathrm{~m}$ which is an error of $0.9 \%$.
In contrast, backward numerical integration estimates the truck at $-8.0 \mathrm{~m}$, which corresponds to a $5.3 \%$ error. Similar results are observed for the predicted truck velocity. One drawback of the forward and backward Kalman filtering approach is the stepwise discontinuity in the position and velocity trajectories at locations where the PVDF position and velocity data is utilized. However, fixed-interval smoothing successfully averages the forward and backward Kalman filtered position and velocity data as shown in figure 12 .

\subsection{Time-synchronized vehicle-bridge response}

During dynamic testing of the Geumdang Bridge, the wireless monitoring system proves reliable in its data collection functionality. When the truck is kept within $700 \mathrm{~m}$ of the wireless monitoring system base station, a data delivery rate of $100 \%$ is achieved. Outside of $700 \mathrm{~m}$, communication between the system base station and the truck-based wireless sensor nodes experiences some intermittent data losses. A total of 


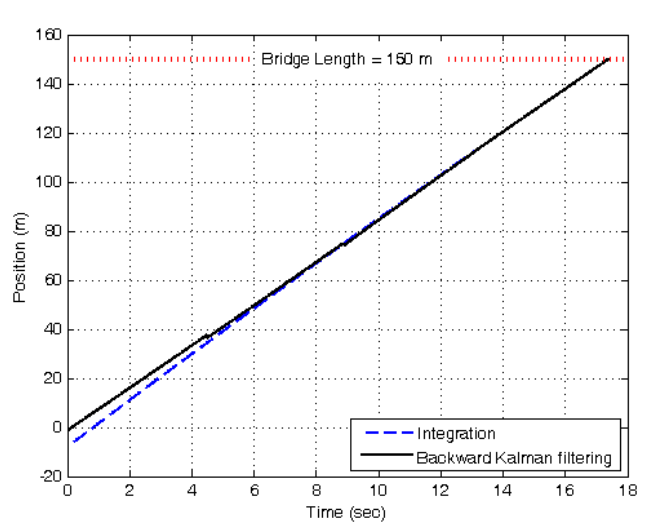

(a)
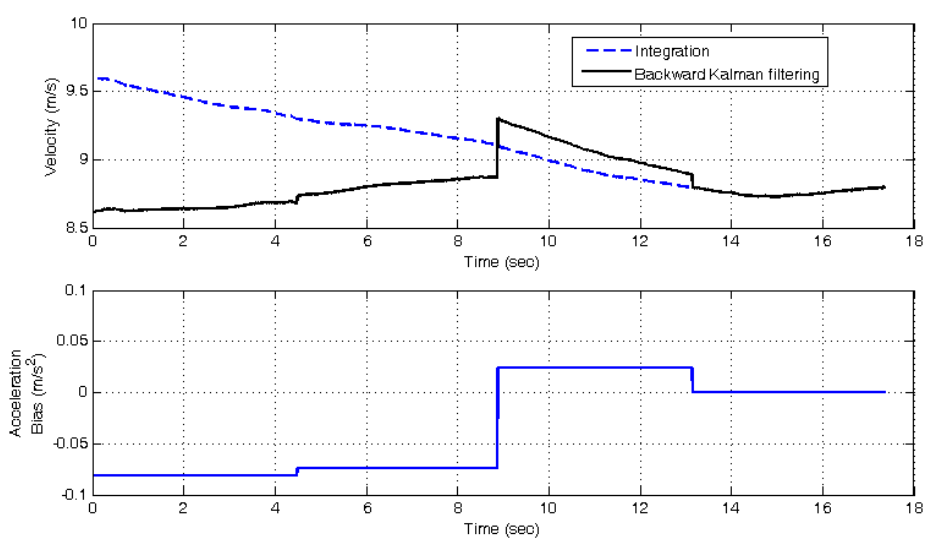

(b)

Figure 11. Results from the backward Kalman filter: (a) estimated truck trajectory along the $150 \mathrm{~m}$ bridge and (b) estimated velocity (top) and estimated accelerometer bias (bottom).
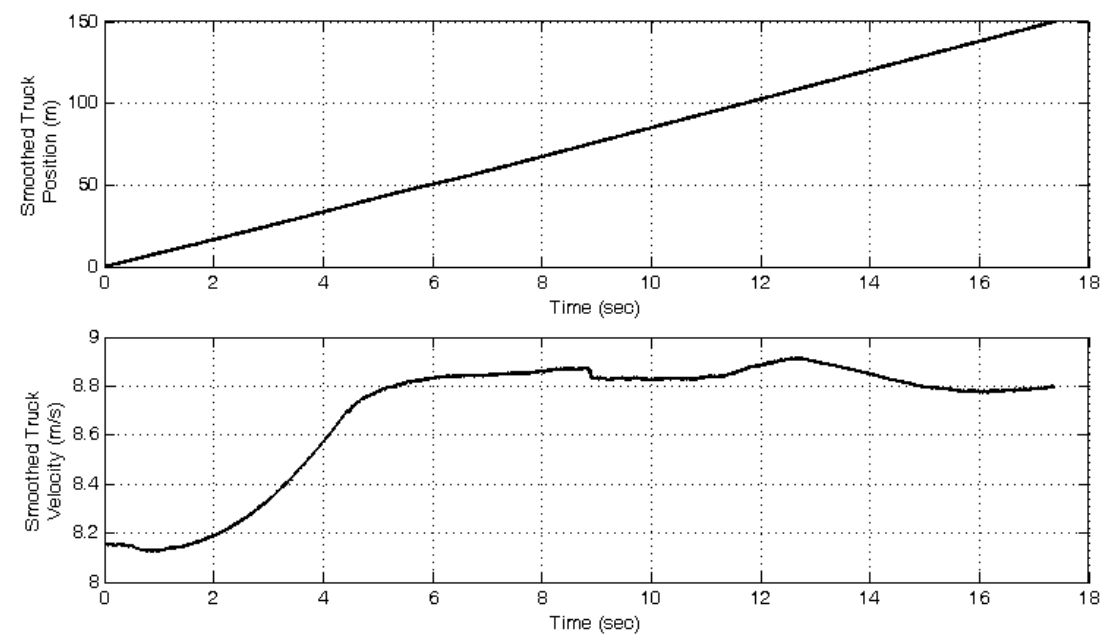

Figure 12. Final estimated truck position (top) and velocity (bottom) based on fixed-interval smoothing.

18 tests are conducted on the bridge with the truck driven at different velocities over the bridge (ranging from 30 to $65 \mathrm{~km} \mathrm{~h}^{-1}$ ). For example, the vertical acceleration response of the truck when driving at $30 \mathrm{~km} \mathrm{~h}^{-1}$ is shown in figure 13 . Based on the estimated truck trajectory, the point in time when each of the truck axles drives over the bridge expansion joints is superimposed on the truck response plots. The truck body and individual axles all experience significant vertical accelerations when driving over the expansion joints. However, the vertical acceleration response of the truck body and axles is damped out quickly by the truck suspension system. Asides from the large vertical accelerations experienced when driving over the expansion joints, smaller vertical accelerations are observed at each of the axles when the truck is driving over the spans. These accelerations are suspected to result from the interaction of the vehicle with the road surface (e.g. due to road roughness) and with the bridge itself.

For the same test (i.e. truck crossing at $30 \mathrm{~km} \mathrm{~h}^{-1}$ ), the corresponding response of the bridge is presented in figure 14 . The vertical acceleration response of the Geumdang Bridge is strongly influenced by the location of the truck relative to the individual spans of the bridge. In figure 14(a), the acceleration measured by sensors \#1-5 (see figure 3), which are installed along the centerline of the first independent span, clearly show the global response of the span when the truck is on that particular span. This low-frequency response corresponds to the global dynamic response of the girders. It is likely some small-amplitude, high-frequency localized response of the bridge deck (acting as a dynamic diaphragm) is also present in the acceleration response. In figure 14(b), the truck has crossed into the second independent span resulting in strong vertical accelerations in sensors \#6-10. When the truck is on the second span, only minor small-amplitude vibrations are observed on the first and third spans. The third span of the Geumdang Bridge is a more complicated span with a pier situated at the span mid-point; this results a more complex bridge response. In figure $14(\mathrm{c})$, the truck is on the third span but to the north side of the center pier. This results in high-amplitude accelerations with higher-frequency content for sensors \#11-15. However, figure 14(d) reveals sensors \#16-20 (which are on the south side of the center pier) also experience high-amplitude accelerations but characterized by lower-frequency content. The opposite situation occurs when the truck moves to the south side of the central pier. This complex dynamic behavior is due to the dynamic coupling of the vehicle and bridge. 

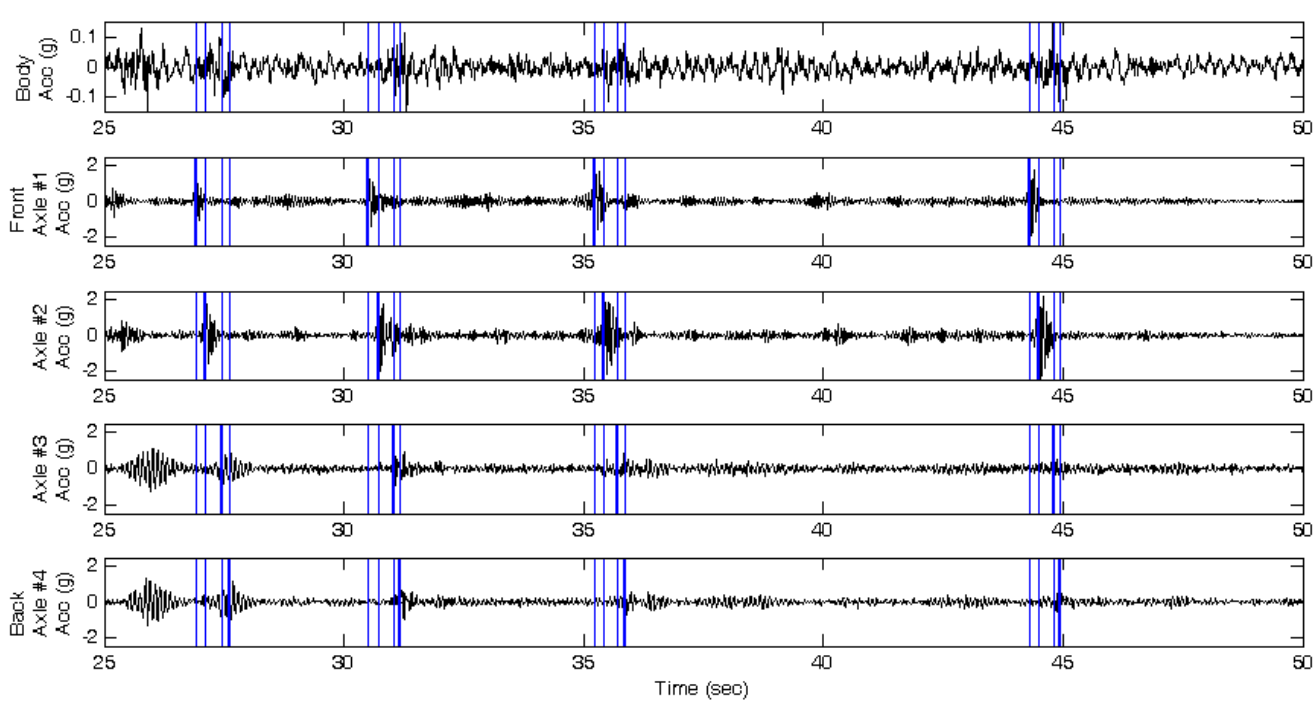

Figure 13. Measured truck vertical acceleration response when driven at $30 \mathrm{~km} \mathrm{~h}^{-1}$ over the Geumdang Bridge. Vertical lines denote the time when each axle crosses a bridge expansion joint. The thicker of the four vertical lines denotes the time of crossing of that specific instrumented axle.
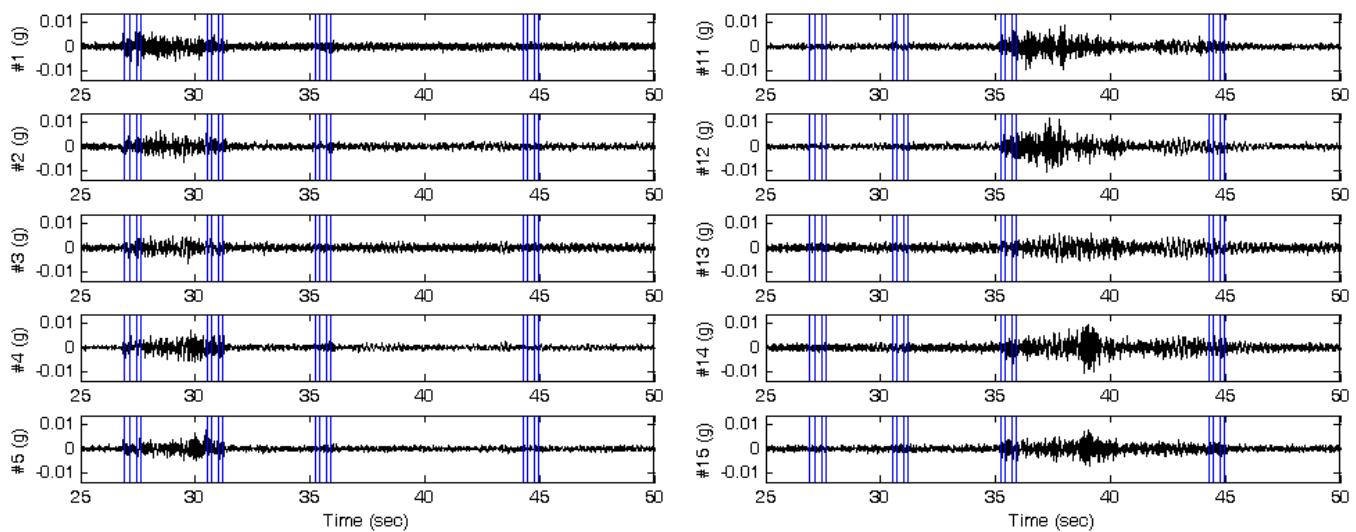

(a)

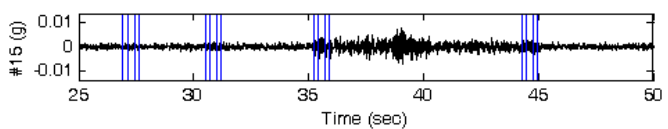

(b)
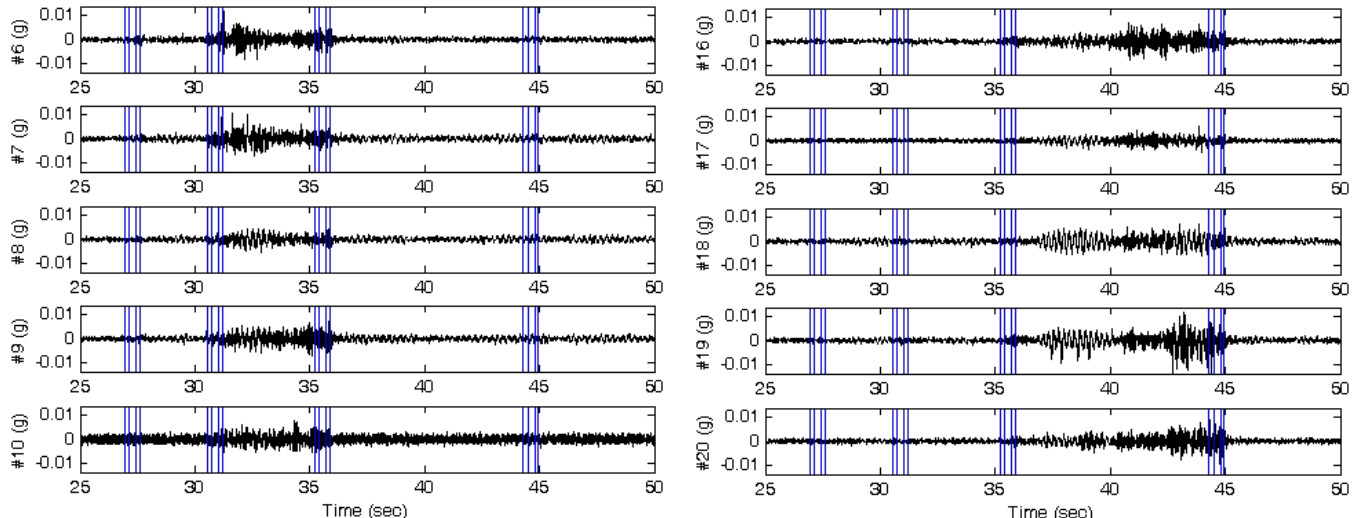

(c)

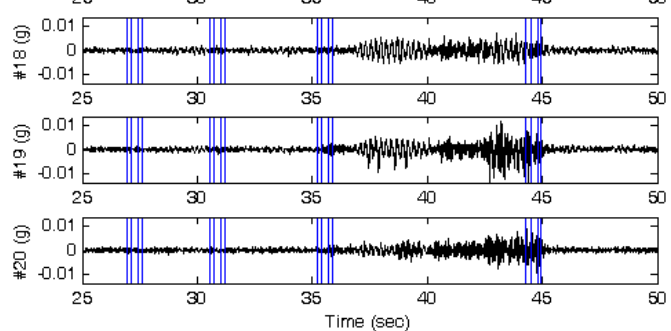

(d)

Figure 14. Measured vertical acceleration response of the Geumdang Bridge during the $30 \mathrm{~km} \mathrm{~h}^{-1}$ truck run: (a) sensors \#1-5 on span 1 (30 m long); (b) sensors \#6-10 on span 2 (40 m long); (c) sensors \#11-15 on the first part of span 3 (40 m long); (d) sensors \#16-20 on the second part of span 3 (40 m long). Vertical lines denote time when each axle crosses a bridge expansion joint.

\section{Conclusions}

In this study, vehicle-bridge interaction is successfully observed using a comprehensive wireless monitoring system capable of unifying data acquisition from mobile wireless sensors on a truck with permanent wireless sensors installed on a bridge. The wireless vehicle-bridge monitoring system is scalable, low cost and proven to be reliable during dynamic testing. In this study, the Geumdang Bridge in Icheon, Korea is selected for dynamic testing using a 20.9 ton truck. Installed 
along the $150 \mathrm{~m}$ northern span of the bridge is a wireless sensor network with 20 wireless vertical accelerometers and 5 wireless PVDF tactile sensors to measure the bridge response and the location of the truck. Similarly, the truck is instrumented with five wireless vertical accelerometers, one wireless horizontal accelerometer and one wireless gyroscope. The 32 wireless sensor nodes are time-synchronized by the wireless monitoring system base station installed on the bridge. The system proved reliable with $100 \%$ data delivery when the truck was within $700 \mathrm{~m}$ of the base station. Using a Kalman filter combined with fixed-interval smoothing, the trajectory of the truck is accurately identified using the horizontal truck acceleration and the PVDF tactile sensor outputs. Furthermore, the time-history data collected from the vehicle and bridge revealed the dynamic coupling that exists between the vehicle and the bridge. By exploring the time-synchronized vibration data of the position-identified vehicle and the bridge, the dominant sources of vehicle-bridge interaction are found experimentally. The main source of vehicle-bridge interaction is the road roughness (e.g. the irregularity of the deck surface) and the sudden change in the pavement topology (e.g. the existence of an expansion joint). Two dominant bridge vibration responses are induced by the moving, vertically vibrating vehicle: low-frequency global structure dynamic responses and high-frequency member-level localized responses (e.g. diaphragm vibrations).

The experimental findings of this study will ultimately help researchers in the smart structure field by offering a scalable means of monitoring both structural demand (i.e. truck loads) and capacity (i.e. bridge responses) in bridge structures. Future work is aimed towards generalizing the wireless monitoring system architecture to facilitate more ad hoc connectivity between the mobile wireless sensors and the bridge wireless sensors. Also, efforts are underway to formulate a detailed analytical model using truckbridge response data that accurately captures vehicle-bridge interaction phenomena. Specifically, a two-stage system identification approach is currently under exploration by the authors [10]. This approach will first utilize the free-vibration response of the bridge to create an output-only model of the bridge system followed by stochastic subspace identification using truck-bridge response data to extract the truck load (i.e. position and magnitude). With an accurate estimation of the bridge load, a structural health monitoring system would be empowered with a quantifiable load history that can be correlated to the long-term deterioration of the bridge. For example, the monitoring system could monitor truck loads over the complete life of the bridge to derive the cumulative load effect. Based on the measured bridge response history coupled with the measured cumulative load effect, the overall reliability of the bridge system could be easily estimated using a safety index, $\beta$, as offered by first-order reliability methods (FORM).

\section{Acknowledgments}

This work is supported by the National Science Foundation under grant CMMI-0726812 (Program Director Dr S C Liu). Additional support was provided by the NIST Technology
Innovation Program (contract 70NANB9H9008), Korea Research Foundation Grant funded by the Korean Government (MOEHRD) (KRF-2007-612-D00136) and the Smart InfraStructure Technology Center (SISTeC) at KAIST. The authors would also like to thank Professor Chung-Bang Yun (KAIST) for his support and encouragement.

\section{References}

[1] AASHTO 2002 Standard Specifications for Highway Bridges (Washington DC: American Association of State Highway and Transportation Officials)

[2] Ansari F 2007 Practical implementation of optical fiber sensors in civil structural health monitoring J. Intell. Mater. Syst. Struct. 18 879-89

[3] Chen Y, Feng M Q and Tan C-A 2009 Bridge structural condition assessment based on vibration and traffic monitoring J. Eng. Mech. 135 747-58

[4] Fraser M, Elgamal A, Xianfei H and Conte J P 2010 Sensor network for structural health monitoring of a highway bridge J. Comput. Civ. Eng. 24 11-24

[5] Gillespie T K and Karamihas S M 2000 Simplified models for truck dynamic response to road inputs Int. J. Heavy Veh. Syst. 7 52-63

[6] Green M F and Cebon D 1994 Dynamic response of highway bridges to heavy truck loads: theory and experimental validation J. Sound Vib. 170 51-78

[7] Greve D W, Sohn H, Yue C P and Oppenheim I J 2007 An inductively coupled Lamb wave transducer IEEE Sens. J. 7 295-301

[8] Hwang E-S and Nowak A S 1991 Simulation of dynamic load for bridges J. Struct. Eng. 117 1413-34

[9] Jang S, Jo H, Cho S, Mechitov K, Rice J A, Sim S-H, Jung H-J, Yun C-B, Spencer B F S and Agha G 2010 Structural health monitoring of a cable-stayed bridge using smart sensor technology: deployment and evaluation Smart Struct. Syst. 6 439-60

[10] Kim J 2011 System identification of civil engineering structures through wireless structural monitoring and subspace system identification methods Civil and Environmental Egineering Department (Ann Arobor, MI: University of Michigan)

[11] Kim J, Swartz R A, Lynch J P, Lee J J and Lee C G 2010 Rapid-to-deploy reconfigurable wireless structural monitoring systems using extended-range wireless sensors Smart Struct. Syst. 6 505-24

[12] Kwasniewski L, Wekezer J, Roufa G, Li H Y, Ducher J and Malachowski J 2006 Experimental evaluation of dynamic effects for a selected highway bridge J. Perform. Constr. Facil. 20 253-60

[13] Lawrence A 1998 Modern Inertial Technology: Navigation, Guidance, and Control (New York: Springer)

[14] Lee C-G, Lee W-T, Yun C-B and Choi J-S 2004 Development of Integrated System for Smart Evaluation of Load Carrying Capacity of Bridges (Daejeon: Korea Advanced Institute of Science and Technology)

[15] Lee H S, Hong Y H and Park H W 2010 Design of an FIR filter for the displacement reconstruction using measured acceleration in low-frequency dominant structures Int. J. Numer. Methods Eng. 82 403-34

[16] Lynch J P, Wang Y, Loh K, Yi J H and Yun C B 2006 Performance monitoring of the Geumdang bridge using a dense network of high-resolution wireless sensors Smart Mater. Struct. 15 1561-75

[17] Mascarenas D, Flynn E, Farrar C, Park G and Todd M 2009 A mobile host approach for wireless powering and interrogation of structural health monitoring sensor networks IEEE Sens. J. 9 1719-26 
[18] Mizuno Y, Fujino Y, Kataoka K and Matsumoto Y 2008 Development of a mobile sensing unit and its prototype implementation Tsinghua Sci. Technol. $13223-7$

[19] Oppenheim I J 2003 Two demonstrations of MEMS in civil engineering J. Aerosp. Eng. 1699

[20] Ozevin D, Greve D W, Oppenheim I J and Pessiki S P 2006 Resonant capacitive MEMS acoustic emission transducers Smart Mater. Struct. 15 1863-71

[21] Pakzad S N, Fenves G L, Kim S and Culler D E 2008 Design and implementation of scalable wireless sensor network for structural monitoring J. Infrastruct. Syst. 14 89-101

[22] Ryu J 2004 State and parameter estimation for vehicle dynamics control using GPS Department of Mechanical Engineering (Stanford, CA: Stanford University)

[23] Salas K I and Cesnik C E S 2010 Guided wave structural health monitoring using CLoVER transducers in composite materials Smart Mater. Struct. 19015014

[24] Simon D 2006 Optimal State Estimation (Hoboken, NJ: Wiley)

[25] Smyth A and Wu M 2007 Multi-rate Kalman filtering for the data fusion of displacement and acceleration response measurements in dynaminc system monitoring Mech. Syst. Signal Process. 21 706-23

[26] Stengel R F 1994 Optimal Control and Estimation (New York: Dover)

[27] Suzhen L and Zhishen W 2007 Development of distributed long-gage fiber optic sensing system for structural health monitoring Struct. Health Monitor. 6 133-43
[28] Swartz R A, Jung D, Lynch J P, Wang Y, Shi D and Flynn M P 2005 Design of a wireless sensor for scalable distributed in-network computation in a structural health monitoring system 5th Int. Workshop on Structural Health Monitoring (Stanford, CA) pp 1570-7

[29] Swartz R A, Lynch J P, Zerbst S, Sweetman B and Rolfes R 2010 Structural monitoring of wind turbines using wireless sensor networks Smart Struct. Syst. 6 183-96

[30] Swartz R A, Zimmerman A T, Lynch J P, Rosario J, Brady T, Salvino L and Law K H 2011 Hybrid wireless hull monitoring system for naval combat vessels Structure and Infrastructure Engineering at press

[31] Szary P and Maher A 2009 Implementation of Weigh-in-Motion (WIM) Systems (Washington, DC: Federal Highway Administration)

[32] Whelan M J and Janoyan K D 2009 Design of a robust, high-rate wireless sensor network for static and dynamic structural monitoring J. Intell. Mater. Syst. Struct. 20 849-63

[33] Yun C B, Sohn H, Koo K Y, Wang M L, Zhang Y and Lynch J P 2009 US-Korea collaborative research for bridge monitoring testbeds (ICOSSAR): Proc. Int. Conf. on Structural Safety and Reliability (Osaka)

[34] Zarchan P, Musoff M and Lu F K 2009 Fundamentals of Kalman Filtering: A Practical Approach American Institute of Aeronautics \& Astronauts 